OPEN ACCESS

Edited by:

Brad Day,

Michigan State University,

United States

Reviewed by:

Matias Pasquali,

University of Milan, Italy

Sébastien Bontemps-Gallo,

Institut Pasteur de Lille, France

Malgorzata Golanowska

Independent Researcher, Heerlen,

Netherlands

*Correspondence:

Lian-Hui Zhang

Inzhang01@scau.edu.cn

Specialty section:

This article was submitted to

Plant Microbe Interactions,

a section of the journal

Frontiers in Microbiology

Received: 15 June 2019 Accepted: 08 August 2019

Published: 21 August 2019

Citation:

Shi Z, Wang $Q, L i Y$, Liang Z, Xu L, Zhou J, Cui Z and Zhang L-H (2019)

Putrescine Is an Intraspecies

and Interkingdom Cell-Cell

Communication Signal Modulating

the Virulence of Dickeya zeae.

Front. Microbiol. 10:1950.

doi: 10.3389/fmicb.2019.01950

\section{Putrescine Is an Intraspecies and Interkingdom Cell-Cell Communication Signal Modulating the Virulence of Dickeya zeae}

\author{
Zurong Shi',2,3, Qingwei Wang'2, Yasheng Li1,2, Zhibing Liang ${ }^{1,2}$, Linghui $X u^{2}$, \\ Jianuan Zhou ${ }^{1,2}$, Zining Cui ${ }^{1,2}$ and Lian-Hui Zhang ${ }^{1,2 *}$ \\ 1 Guangdong Province Key Laboratory of Microbial Signals and Disease Control, South China Agricultural University, \\ Guangzhou, China, ${ }^{2}$ Integrative Microbiology Research Centre, South China Agricultural University, Guangzhou, China, \\ ${ }^{3}$ College of Agriculture and Biology, Zhongkai University of Agricluture and Engineering, Guangzhou, China
}

The infections caused by Dickeya zeae become a severe problem in recent years, but the regulatory mechanisms that govern the bacterial virulence remain to be fragmental. Here we report the investigation of potential involvement of polyamines in regulation of $D$. zeae virulence. We showed that null mutation of speA encoding arginine decarboxylase dramatically decreased the bacterial swimming motility, swarming motility and biofilm formation, and exogenous addition of putrescine effectively rescues the defective phenotypes of $D$. zeae. HPLC and mass spectrometry analysis validated that speA was essential for production of putrescine in $D$. zeae. In addition, we demonstrated that D. zeae EC1 could detect and response to putrescine molecules produced by itself or from host plant through specific transporters. Among the two transporters identified, the one represented by PotF played a dominated role over the other represented by $\mathrm{PlaP}$ in modulation of putrescine-dependent biological functions. Furthermore, we provided evidence that putrescine signal is critical for D. zeae EC1 bacterial invasion and virulence against rice seeds. Our data depict a novel function of putrescine signal in pathogen-host communication and in modulation of the virulence of an important plant bacterial pathogen.

Keywords: putrescine, cell-cell signaling, pathogen-host communication, polyamine, regulatory elements, regulatory network

\section{INTRODUCTION}

Dickeya zeae, the causative agents of maize stalk rot and rice foot rot diseases, is previously named as Erwinia chrysanthemi pv. zeae (Sinha and Prasad, 1977; Goto, 1979; Nassar et al., 1994; Hussain et al., 2008). More recently, D. zeae was also shown to cause severe bacterial soft rot disease in banana, which is an important economical crop in many tropical and subtropical countries and regions (Zhang et al., 2013). D. zeae is much less studied compared with other well characterized species of the Dickeya genus, such as D. dadantii, formally known as Erwinia chrysanthemi, which are known to deploy a range of virulence determinants to enhance its competitive advantages against its host (Barras et al., 1994; Collmer and Bauer, 1994). In recent years, we found that D. zeae strain EC1 produces two polyketide phytotoxins, known as zeamine and zeamine II, which are 
able to inhibit rice seed germination (Zhou et al., 2011; Cheng et al., 2013). Deletion of the genes encoding zeamine and zeamine II production abrogated and attenuated the bacterial virulence, respectively (Zhou et al., 2011; Cheng et al., 2013), suggesting that zeamines are the key virulence determinants of $D$. zeae. In addition, zeamines are also potent antibiotics, which effectively kill a wide range of bacterial and fungal pathogens at low end of micromolar concentrations (Wu et al., 2010; Liao et al., 2014). In addition to phytotoxin zeamines, evidence suggests that other virulence associated traits might also contribute to the virulence of $D$. zeae. The acyl-homoserine lactone (AHL) quorum sensing (QS) system is widely conserved in Gramnegative bacteria, including $D$. zeae. The AHL QS system is consist of a luxI homolog, which encodes for AHL signal biosynthesis, and a $\operatorname{luxR}$ homolog, which encodes an AHLdependent transcription factor. D. zeae has only one set of AHL QS system with expI, the homolog of luxI, encoding for biosynthesis of the QS signal $N$-(3-oxo-hexanoyl)-homoserine lactone (OHHL) (Hussain et al., 2008). Disruption of expI had little effect on zeamines production in a rich medium, but resulted in increased bacterial cell motility, decreased production of extracellular enzymes, and partially attenuated bacterial virulence against dicotyledonous plant Chinese cabbage and monocotyledonous plant rice (Hussain et al., 2008).

Evidence suggests that the AHL QS system may regulate the virulence genes through the transcription factor SlyA. Genetic analysis showed that SlyA positively regulates biofilm formation, virulence on rice seeds and negatively controls bacterial motility, and in trans expression of sly $A$ in the expI mutant restored the phenotypes of bacterial motility and biofilm formation (Zhou et al., 2016), suggesting that SlyA may act at the downstream of the AHL QS system. In contrast to the AHL QS system that has only a minor effect on zeamines biosynthesis, null mutation of sly $A$ led to a significant reduction in zeamines production (Zhou et al., 2016). The findings suggest that the AHL system may only partially influence the biological functions of SlyA, indicating the complexity of virulence regulatory mechanisms in D. zeae.

Spermidine, spermine, and putrescine constitute a group of ubiquitous aliphatic small polycationic molecules known as polyamines, which are widely distributed from bacteria to plants and animals. The evidence of polyamine molecules as signaling molecules in microorganisms has been accumulating in recent years. We reported previously that spermidine and spermine from mammalian host are the interkingdom cellcell communication signals, which are taken up through a potent ABC transporter SpuBCDEF and specifically induce the transcriptional expression of the genes encoding the type III secretion system in a human bacterial pathogen Pseudomonas aeruginosa (Zhou et al., 2007; Wu et al., 2012). In another human pathogen Proteus mirabilis, it is putrescine that acts as an extracellular signal required for bacterial swarming motility and invasion ability (Sturgill and Rather, 2004; Kurihara et al., 2013). Putrescine has also been known for its role in modulation of biofilm formation and disassembly by human pathogen Yersinia pestis and environmental bacterium Shewanella oneidensis, respectively (Patel et al., 2006; Ding et al., 2014). Additionally, evidence indicates that putrescine produced by the plant pathogen Ralstonia solanacearum might also act on host to accelerate plant wilt disease (Lowe-Power et al., 2018). Putrescine biosynthesis in bacteria has two alternative pathways, one pathway starts with ornithine by ornithine decarboxylase (SpeC) known as ODC pathway (Boyle et al., 1984). The second pathway involves the sequential conversion from arginine by arginine decarboxylase (SpeA) and agmatinase (SpeB) to putrescine (Boyle et al., 1984). The triamine spermidine is formed from putrescine by addition an aminopropyl group derived from decarboxylated $S$-adenosylmethionine by the spermidine synthase encoded by speE (Tabor et al., 1986; Tabor and Tabor, 1987).

To test whether polyamines could also play a role in regulation of $D$. zeae virulence, we conducted a systemic deletion analysis on the genes encoding polyamine biosynthesis and transport. Our results showed that deletion of the arginine decarboxylase gene speA for putrescine biosynthesis could drastically change the patterns of bacterial motility and biofilm formation. We also found two putrescine specific transporters that collectively play a critical role for bacterial response to putrescine signals produced by bacteria or host plant in regulation of $D$. zeae motility and virulence. To our knowledge, this is the first report on modulation of bacterial motility by putrescine signals from eukaryotic cells.

\section{MATERIALS AND METHODS}

\section{Bacterial Strains, Plasmids, and Reagents}

The bacterial strains and plasmids used in this study are listed in Supplementary Table S1. D. zeae EC1 and its derivatives were grown at $28^{\circ} \mathrm{C}$ in $\mathrm{LB}$ medium unless otherwise stated. Minimal medium $\left(\mathrm{K}_{2} \mathrm{HPO}_{4} 10.5 \mathrm{~g} / \mathrm{L}, \mathrm{KH}_{2} \mathrm{PO}_{4} 4.5 \mathrm{~g} / \mathrm{L},(\mathrm{NH})_{2} \mathrm{SO}_{4} 2 \mathrm{~g} / \mathrm{L}\right.$, $\mathrm{MgSO}_{4} .7 \mathrm{H}_{2} \mathrm{O} 0.2 \mathrm{~g} / \mathrm{L}, \mathrm{FeSO}_{4} 0.005 \mathrm{~g} / \mathrm{L}, \mathrm{CaCl}_{2} 0.01 \mathrm{~g} / \mathrm{L}, \mathrm{MnCL}_{2}$ $0.002 \mathrm{~g} / \mathrm{L}$, glycerol $2 \mathrm{~g} / \mathrm{L}$, and mannitol $2 \mathrm{~g} / \mathrm{L}$ ) was used for comparison of bacterial growth rate. Putrescine, spermidine, and spermine were purchased from Sigma-Aldrich. Antibiotics were added at the following final concentrations when required: kanamycin (Km), $100 \mu \mathrm{g} / \mathrm{mL}$; streptomycin (Str), $50 \mu \mathrm{g} / \mathrm{mL}$; gentamycin $(\mathrm{Gm}), 50 \mu \mathrm{g} / \mathrm{mL}$; and tetracycline $(\mathrm{Tc}), 10 \mu \mathrm{g} / \mathrm{mL}$.

\section{Generation of In-Frame Deletion Mutants and Complementation}

The in-frame deletion mutants of speA, speC, speE, potF, and plaP were generated through homologous recombination as previously described (Zhou et al., 2011; Cheng et al., 2013), and named as $\Delta s p e A, \Delta s p e C, \Delta s p e E, \Delta p o t F$, and $\Delta p l a P$, respectively. The speA/potF and speA/plaP double deletion mutants were generated by deleting pot $F$ and $p l a P$ in the genetic background of the speA deletion mutant and designated as $\triangle s p e A \Delta p o t F$ and $\Delta$ speA $\Delta$ plaP, respectively. The speA/potF/plaP triple gene deletion mutant $\triangle$ speA $\triangle$ potF $\triangle$ plaP was generated by deleting plaP using the double mutant $\Delta s p e A \Delta p o t F$ as the parental strain. The detailed descriptions of strains and mutants were provided in Supplementary Table S1. All the primers used in this study were listed in Supplementary Table S2. Fragments containing 
about 500 bps upstream and downstream regions of target genes were amplified from the chromosomal DNA sample of $D$. zeae strain EC1, respectively, which were linked by second round PCR with the forward primer of the upstream fragment and the reverse primer of the downstream fragment. The fusion PCR fragments were digested with restriction enzymes and ligated to the vector pKNG101 digested with the same enzymes. The resultant constructs were transformed into Escherichia coli CC118. D. zeae mutants were generated by tri-parental mating following the protocol described previously (Cheng et al., 2013).

For complementation analysis, the encoding regions of genes $s p e A, p o t F$, and plaP were amplified by the primers listed in Supplementary Table S2. The PCR products were digested with restriction enzymes and then cloned into the expression vector pBBR1-MCS4 digested with the same enzymes. The complementation constructs were introduced into corresponding mutants by tri-parental mating and confirmed by PCR analysis.

\section{Bacterial Motility and Biofilm Assays}

To examine changes in bacterial swimming motility, $2 \mu \mathrm{L}$ overnight cultures of $D$. zeae strains were spotted inside the agar at the center of semisolid Bacto tryptone agar medium (per liter contains $10 \mathrm{~g}$ bacteriological peptone, $5 \mathrm{~g} \mathrm{NaCl}, 3 \mathrm{~g}$ agar). After incubation for $24 \mathrm{~h}$ at $28^{\circ} \mathrm{C}$, the diameters of swimming motility were measured. The swarming motility was assayed under the same conditions, except that bacterial cells were spotted onto the semisolid medium containing (per liter) $5 \mathrm{~g}$ tryptone, $5 \mathrm{~g}$ $\mathrm{NaCl}$, and $4 \mathrm{~g}$ agarose. The experiments were repeated three times in triplicates.

For measurement of biofilm formation, $1 \mu \mathrm{L}$ overnight cultures of D. zeae strain EC1 and its derivatives were added to a 96-well plate containing $99 \mu \mathrm{L}$ fresh SOBG medium (tryptone, $20 \mathrm{~g} / \mathrm{L}$, yeast extract, $5 \mathrm{~g} / \mathrm{L}, \mathrm{MgSO} 4.7 \mathrm{H}_{2} \mathrm{O} 2.4 \mathrm{~g} / \mathrm{L}, \mathrm{NaCl} 0.5 \mathrm{~g} / \mathrm{L}$, $\mathrm{KCl} 0.186 \mathrm{~g} / \mathrm{L}$, glycerol $20 \mathrm{~mL} / \mathrm{L}$ ). The plates were incubated at $28{ }^{\circ} \mathrm{C}$ with shaking at $150 \mathrm{rpm}$ for $18 \mathrm{~h}$ and the liquid cultures were removed. The attached bacterial cells were incubated with $0.1 \%$ crystal violet (CV) for 15 min before draining the liquid and washing three times with water. CV molecules retained by attached bacterial cells were solubilized with 95\% ethanol. The amount of dye bound, representing the mass of attached bacterial cells, was monitored by measuring the absorbance at $570 \mathrm{~nm}$ on a microplate spectrophotometer. The experiments were repeated three times with triplicates.

\section{Chemical Complementation With Exogenous Polyamines}

Different concentrations of putrescine and spermidine were added to semisolid medium as indicated for determination of their activity in restoration of swimming motility phenotype of the mutant $\Delta s p e A$. Swimming motility of the D. zeae strains were quantified using the method described above. To rescue the swarming motility and biofilm formation phenotypes of the mutant $\Delta$ speA, putrescine and spermidine were added to corresponding media at a final concentration of $0.1 \mathrm{mM}$, respectively. The experiments were repeated three times with triplicates.

\section{Bacterial Sample Preparation and Polyamine Derivatization}

The cellular levels of putrescine in wild type strain EC1 and mutant $\triangle$ speA cells were measured based on the methods described previously with minor modifications (Kashiwagi and Igarashi, 1988; Kurihara et al., 2008; Lee et al., 2009; Sethi et al., 2011). Briefly, bacterial cells were grown to an $\mathrm{OD}_{600}$ of 1.7 at $28^{\circ} \mathrm{C}$ with shaking at $200 \mathrm{rpm}$ in minimal medium, then pelleted and the wet weight of bacterial cells was recorded. Cell pellets were washed with $\mathrm{ddH}_{2} \mathrm{O}$ for three times and resuspended with $600 \mu \mathrm{L}$ lysis buffer (20 mM MOPS, $\mathrm{pH}$ 8.0, $10 \mathrm{mM} \mathrm{NaCl}, 4 \mathrm{mM} \mathrm{MgCl}_{2}$ ), then lysed by three cycles of freeze/thawing with liquid nitrogen. Trichloroacetic acid (40\%) was added to the lysed cell suspension at a final concentration of $10 \%$, and the mixtures were incubated on ice for $30 \mathrm{~min}$. The supernatants were collected by centrifugation and used for derivatization. For each sample $(500 \mu \mathrm{L})$, derivatization was carried out by adding $125 \mu \mathrm{L}$ of $2 \mathrm{M} \mathrm{NaOH}$ and $100 \mu \mathrm{L}$ of benzoyl chloride and incubated for $30 \mathrm{~min}$ at room temperature with constant shaking (Sethi et al., 2011). Benzoylated putrescine was used for analysis with high performance liquid chromatography mass spectrometry (LC-MS). The derivatized samples were separated by using an Eclipse Plus C18 (Aglent) column fitted with a 100 - by $2.1 \mathrm{~mm}$ guard column with a flow rate of $0.3 \mathrm{~mL} / \mathrm{min}$. Mass spectroscopy (Aglent 6540B Q-TOF) was used to verify the identity of each peak observed in HPLC fractions. The experiments were repeated three times in triplicates. A standard curve was generated by using various concentrations of benzoylated putrescine in duplicates.

\section{Culture With Rice Seedling Extract}

Seeds of rice variety $\mathrm{CO} 39$ were soaked with water at room temperature for $24 \mathrm{~h}$, transferred onto moistened gauze in a plastic sorting box and were incubated at $28^{\circ} \mathrm{C}$ with a $16 \mathrm{~h}$ light and $8 \mathrm{~h}$ dark cycle for 3 days. About $3 \mathrm{~g}$ seedlings were placed in mortar and grinded with liquid nitrogen. The powders of seedlings were homogenated in $30 \mathrm{~mL} \mathrm{ddH_{2 }} \mathrm{O}$ and stored at $4^{\circ} \mathrm{C}$ for $24 \mathrm{~h}$ to extract free polyamines, then centrifuged at $8,000 \mathrm{rpm}$ for $15 \mathrm{~min}$ and supernatants were collected. The sterilized supernatants were mixed with the semi-preparative swimming medium at the ratio of 1:5. The semi-preparative swimming medium was added with the same proportion of sterilized water as blank control. Swimming motility was measured as described above. The rice seedling extracts were also taken for polyamines analysis with LC-MS as described above. The experiments were repeated three times in triplicates.

\section{Transcriptome and RT-PCR Analyses}

Overnight cultures of strain EC1 and $\triangle s p e A$ were cultured in swimming motility medium with putrescine at the final concentration of $0.1 \mathrm{mM}$ or without at $28^{\circ} \mathrm{C}$ to $\mathrm{OD}_{600}=0.6$. The RNA samples were prepared using the SV total RNA isolated system kit (Promega) and further purified using the RNA clean kit (Qiagen). The total RNA samples were treated with DNase I for degrading DNA. The mRNA samples were purified from total RNA using poly-T oligo-attached magnetic beads and 
fragmented into short fragments by mixing with fragmentation buffer. Then cDNA fragments were synthesized using random hexamer-primers, dNTPs, RNase $\mathrm{H}$, and DNA polymerase I. The ends of the purified double-strand cDNA were repaired by adding adenine before purification of cDNA fragments with a QIAQuick Kit (QIAGEN, Germany). Sequencing adaptors were ligated to the cDNA fragments and PCR amplification was then performed to enrich the fragments. Finally, the library of cDNAs was constructed and sequenced on an Illumina Hisq platform. Sequenced Reads were obtained by base calling using CASAVA software and saved in FASTQ format. Reads were mapped to the whole genome sequence of $D$. zeae EC1 (CP006929.1). The gene expression level was calculated by using RPKM (Reads per Kilobase of Transcript per Million Reads Mapped) method (Wagner et al., 2012) and analyzed using DEseq (Wang et al., 2010). The genes with statistically significant changes in expression (| $\log 2$ Ratio $\mid \geq 1$ and $q \leq 0.05$ ) were randomly selected for verification. Reverse transcriptionpolymerase chain reaction (RT-PCR) was performed by using StarScript II first-strand cDNA synthesis Mix following the manufacturer's protocol (GenStar) with the RT-PCR primers listed in the Supplementary Table S2. The raw data were available under SRA accession number PRJNA516290.

\section{Rice Seed Germination and Bacterial Invasion Assays}

The rice seed germination assay was conducted as previously described (Hussain et al., 2008). Briefly, overnight bacterial cultures were diluted in 10-fold series, and the CFU of each dilution was determined using heterotrophic plate counting assay. Thirty seeds of rice variety CO39 were added to $9 \mathrm{~mL}$ of bacterial dilution and incubated at room temperature for $5 \mathrm{~h}$. The rice seeds were then washed three times with sterilized water and transferred onto two moistened filter papers in a petri dish. The seeds were then incubated at $28^{\circ} \mathrm{C}$ with a $16 \mathrm{~h}$ light and $8 \mathrm{~h}$ dark cycle, and sterilized water was added when necessary. Rice seeds were incubated with same amount of sterilized water as a blank control. The rate of seed germination was determined 5 days after treatment. The experiment was repeated four times.

For analysis of bacterial invasion, the encoding region of $g f p$ was amplified by PCR, and cloned under the control of the lac promoter in the expression vector pLAFR3, which carries a tetracycline resistance gene. The resultant construct was introduced into D. zeae strain EC1 and its derivatives via triparental mating. Overnight cultures of GFP-labeled strains EC1, $\triangle s p e A$ and $\Delta s p e A \Delta p o t F \Delta$ plaP were resuspended and diluted with $\mathrm{ddH}_{2} \mathrm{O}$ to the CFU of $10^{2}$. Sixteen seeds of rice variety CO39 were added to $1 \mathrm{~mL}$ of bacterial dilution and incubated at room temperature for $5 \mathrm{~h}$, and then transferred onto moistened filter papers in plates. Rice seeds were incubated with same amount of sterilized water as a blank control. The seeds were incubated at $28^{\circ} \mathrm{C}$ of $16 \mathrm{~h}$ light $8 \mathrm{~h}$ dark condition for $24 \mathrm{~h}$. The husks of rice seeds were removed with one group containing 8 seeds per treatment being examined under a fluorescence microscope and the other containing same amount of seeds cut into small pieces and resuspended with $1 \mathrm{~mL}$ sterilized water. Samples were diluted accordingly and spread onto LB plates containing tetracycline, which were cultured for $24 \mathrm{~h}$ to count bacterial colony forming unit (CFU). The experiments were repeated three times.

\section{Statistical Analysis}

For analysis of statistical significance, the data were analyzed using GraphPad Prism's $t$-test and $P<0.05$ was considered significant for all experiments.

\section{RESULTS}

\section{Null Mutation of speA Impairs Cell Motility and Biofilm Formation}

Four enzymes are known to be associated with the biosynthesis of polyamines in E. coli, including arginine decarboxylase (SpeA, NCBI accession YP006135334.1), ornithine decarboxylase (SpeC, NCBI accession BAI27252.1), agmatinase (SpeB, NCBI accession BAE77000.1) and spermidine synthase (SpeE, NCBI accession BAB96695.1) (Boyle et al., 1984; Tabor et al., 1986; Tabor and Tabor, 1987). Blast analyses showed that D. zeae strain EC1 contains homologs of speA (NCBI accession WP_016940691.1), speC (NCBI accession WP_016942161.1), and speE (NCBI accession WP_016942102.1) (Zhou et al., 2015). Sequence alignment analysis showed that speA, speC and speE share about 86,72 , and $79 \%$ similarity at the peptide level with their counterparts in E. coli, respectively.

To determine the roles of polyamines in D. zeae physiology and pathogenesis, we constructed the in frame deletion mutants of speA, speC, and speE using D. zeae strain EC1 as a parental strain, and assayed for phenotype changes. Among these three mutants, the speA deletion mutant $\triangle$ speA showed the significantly decreased swimming motility (Figure 1), swarming motility and biofilm formation (Figures 2A,B), compared with the wild type strain EC1 and the complemented strain $\triangle \operatorname{speA}(s p e A)$. However, deletion of $s p e C$ did not cause any statistically significant phenotype changes, while abrogation of speE showed a marginal effect, if any, compared with the wild type strain EC1 (Supplementary Figure S1). Time course analysis found that the mutant $\triangle s p e A$ grew in a similar rate with the wild type strain EC1 and its complemented strain $\triangle$ speA(speA), indicating that the phenotypes of the speA mutant were not caused by reduced growth rate (Supplementary Figure S2). The above data thus suggest that the enzymatic product of SpeA might play a key role in modulation of cell motility and biofilm formation of D. zeae.

\section{Exogenous Addition of Putrescine Rescues Cell Motility and Biofilm Formation}

Given that speA encodes the key enzyme of polyamine biosynthesis pathway in bacteria, and deletion of speA might decrease the intercellular levels of putrescine and spermidine, we set to determine whether exogenous addition of polyamine molecules could rescue the defective phenotypes of the mutant $\Delta s p e A$. As shown in Figure 3, exogenous addition of putrescine 


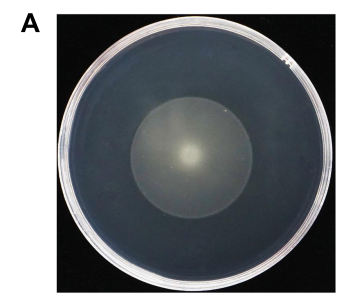

C

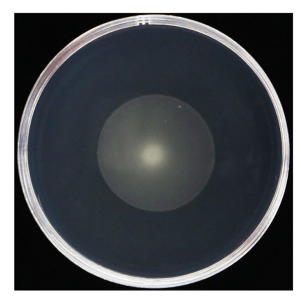

B

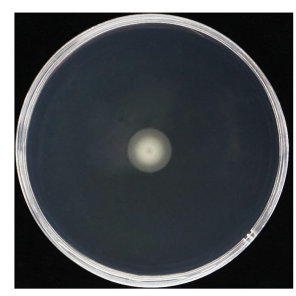

D

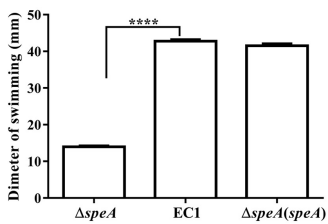

FIGURE 1 | Mutation of the speA gene resulted in reduced swimming motility. (A) D. zeae wild type strain EC1, (B) the speA-disrupted mutant $\Delta s p e A$, (C) strain $\Delta s p e A(s p e A)$ derived by in trans expressing speA in the mutant $\Delta s p e A$. (D) Quantitative measurement of swimming motility of strain EC1 and its derivatives. The photographs were taken after incubation for $24 \mathrm{~h}$ at $28^{\circ} \mathrm{C}$. The data shown are the means $\pm \operatorname{SE}(n=3)$. ${ }^{* * * *} P<0.0001$.

could increase the swimming motility of the mutant $\Delta$ speA in a dosage dependent manner, whereas the effect of spermidine was marginal, and the molecule even inhibited the bacterial swimming motility at a high concentration of $1 \mathrm{mM}$. Similarly, addition of putrescine at a final concentration of $0.1 \mathrm{mM}$ rescued the mutant phenotypes of swarming motility and biofilm formation (Figures 4A,B). Taken together, these data suggest that inactivation of speA might reduce the intracellular putrescine concentration, which led to decreased cell motility and reduced biofilm formation of D. zeae.

\section{Transporters Are Required for Response to Extracellular Putrescine}

Previous studies showed that polyamines molecules are transported into cells through membrane bound transporters. In the well characterized E. coli, four putrescine specific transporters, i.e., PotE, PotFGHI, PuuP, and PlaP, and one spermidine/putrescine transporter PotABCD have been identified and characterized (Furuchi et al., 1991; Kashiwagi et al., 1992; Pistocchi et al., 1993; Kurihara et al., 2009, 2011). Analysis of the D. zeae genome unveiled two putrescine specific transporter loci annotated as potFGHI and plaP, respectively. Amino acid sequence comparison showed that PotF and PlaP from $D$. zeae EC1 share about $81 \%$ identity to the substrate binding proteins PotF and PlaP of E. coli, respectively. Therefore, the potF and plaP genes in D. zeae EC1 are highly likely to encode putrescine binding proteins of the cognate transporter systems. To determine their roles in putrescine transportation, pot $F$ and plaP were deleted in the wild type strain EC1 and the mutant $\triangle$ speA genetic background, respectively. The results showed that single deletion of potF and plaP or deletion of both $p o t F$ and plaP did not affect bacterial swimming motility
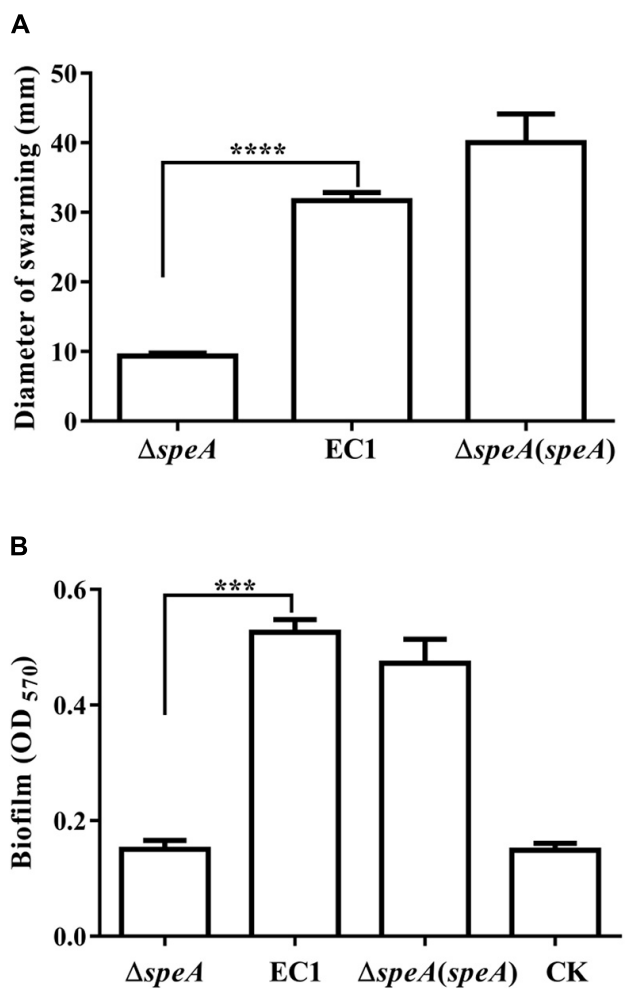

FIGURE 2 | Disruption of speA decreased bacterial swarming motility and biofilm formation. (A) Swarming motility of strain EC1and its derivatives, the diameters were measured after incubation for $18 \mathrm{~h}$ at $28^{\circ} \mathrm{C}$. (B) Biofilm formation of wild type and its derivatives. Bacterial strains were grown in SOBG medium at $28^{\circ} \mathrm{C}$ with shaking at $150 \mathrm{rpm}$ for $18 \mathrm{~h}$, and the medium without inoculation was used as a blank control (CK). The data shown are the means $\pm \operatorname{SE}(n=3)$. ${ }^{* * * *} P<0.0001,{ }^{* * *} P<0.001$.

in comparison with the wild type strain EC1 (Supplementary Figure S3E). We therefore generated the triple-deletion mutant $\Delta$ speA $\Delta$ potF $\triangle$ plaP by deletion of speA/potF/plaP consecutively in the wild type strain $D$. zeae EC1. The results showed that in the swimming plate, the swimming zone of triple-deletion mutant $\Delta$ speA $\Delta$ potF $\Delta$ plaP became smaller than the single deletion mutant $\triangle s p e A$ (Supplementary Figures $\mathbf{S 3 A}, \mathbf{B})$. Interestingly, under the same conditions, while in trans expression of $p o t F$ under the control of a constitutive promoter Plac in $\Delta$ speA $\triangle$ potF $\Delta$ plaP could restore the mutant phenotype (Supplementary Figure S3C), complementation of plaP under the control of same promoter in $\Delta s p e A \Delta p o t F \Delta p l a P$ failed to rescue the mutant motility (Supplementary Figure S3D). This suggests that the transporter PlaP might not be functional when the bacterial cells grown in the swimming plate.

For verification, we generated the double-deletion mutants $\Delta s p e A \Delta p l a P$ and $\Delta s p e A \Delta p o t F$, respectively, and compared their swimming motility with the single-deletion mutant $\triangle s p e A$ and triple-deletion mutant $\triangle$ speA $\Delta$ potF $\triangle$ plaP. The results showed that the swimming motility of the double deletion mutant $\Delta s p e A \Delta p o t F$ and triple-deletion mutant $\Delta s p e A \Delta p o t F \Delta p l a P$ were decreased by about 33 and $35 \%$, respectively, in comparison 

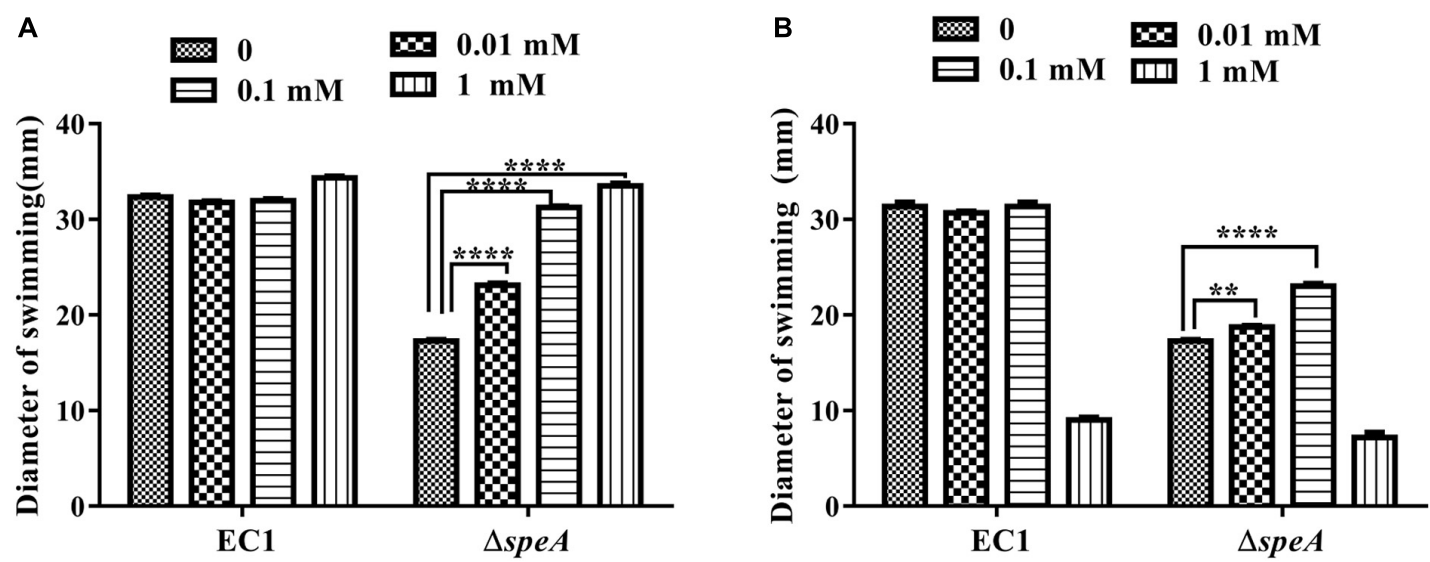

FIGURE 3 | Effect of exogenous addition of polyamines on bacterial swimming motility. Bacterial strains were grown under the same condition but supplemented with different concentrations of putrescine $(\mathbf{A})$ and spermidine (B). The diameters of swimming motility were measured after incubation for $24 \mathrm{~h}$ at $28^{\circ} \mathrm{C}$. The data shown are the means $\pm \mathrm{SE}(n=3)$. $* * * P<0.0001, * * P<0.01$.
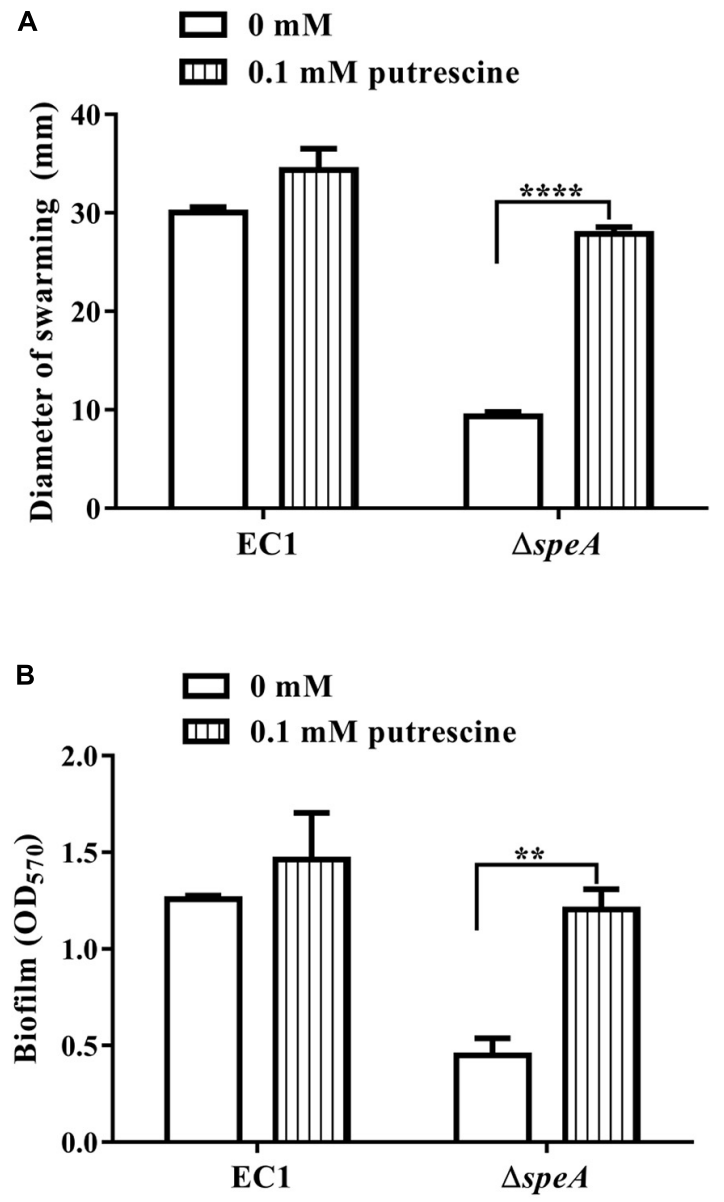
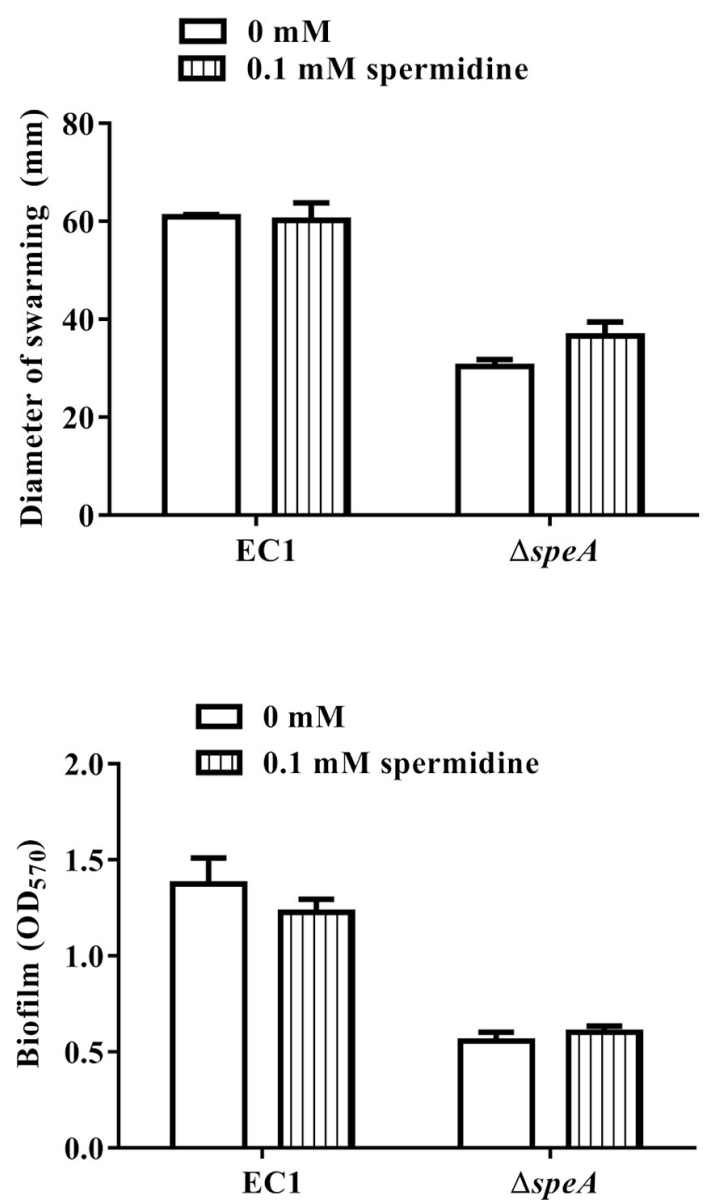

FIGURE 4 | Effect of exogenous addition of putrescine or spermidine on swarming motility and biofilm formation. (A) Swarming motility of EC1 and its derivatives in the presence of $0.1 \mathrm{mM}$ putrescine or spermidine, respectively, the diameter of warming zone was measured at $24 \mathrm{~h}$ after inoculation. (B) Effect of putrescine or spermidine on biofilm formation at a final concentration of $0.1 \mathrm{mM}$. The data shown are the means $\pm \mathrm{SE}(n=3)$. ${ }^{* * * *} P<0.0001,{ }^{* *} P<0.01$. 
with the mutant $\Delta$ speA (Supplementary Figure S3G). However, consistent with the plaP complementation data (Supplementary Figure S3D), the other double deletion mutant $\Delta$ speA $\Delta$ plaP showed a similar swimming motility to its parental strain $\triangle$ speA (Supplementary Figure S3F).

Given that swimming plate might contain only a small amount of putrescine, likely from the medium component bacteriological peptone, we then tested the roles of transporters in the presence of exogenous putrescine. The results showed the swimming motility of the triple-deletion mutant $\Delta$ speA $\Delta$ potF $\Delta$ plaP was much reduced compared with the single deletion mutant $\Delta$ speA (Figures 5A,B). Similar to the complementation experiment without exogenous addition of putrescine (Supplementary Figure S3C), complementation of $\Delta$ speA $\Delta$ potF $\Delta$ plaP with pot $F$ largely restored its swimming motility (Figure 5C). Surprisingly, however, in trans expression of plaP in the mutant $\Delta$ speA $\triangle$ potF $\triangle$ plaP could fully rescue its swimming motility in a capacity better than potF (Figures 5C,D), which differs from the same complementation experiment but without exogenous addition of putrescine (Supplementary Figures S3C,D). Superior performance of PlaP transporter than PotF transporter was further confirmed when the bacterial motilities of speA/potF and speA/plaP double deletion mutants were compared in the presence of exogenous putrescine (Supplementary Figure S3F). Quantitative analysis showed that exogenous addition of putrescine significantly increased the swimming motility of the mutant $\triangle$ speA and the complemented strains $\Delta$ speA $\Delta$ potF $\Delta$ plaP $(p o t F)$ and $\Delta$ speA $\Delta$ potF $\Delta$ plaP $(p l a P)$, whereas the same treatment had no effect on the motility of tripledeletion mutant $\Delta$ speA $\Delta$ potF $\Delta$ plaP (Figure 5E), validating the key role of two transporters in efflux of exogenous putrescine in modulation of bacterial motility.

PotF and PlaP from $D$. zeae share a high similarity with their counterparts in E. coli with over $81 \%$ identity at amino acid level. Among them, PotF was previously demonstrated as a specific putrescine-binding protein (Vassylyev et al., 1998), whereas PlaP is a relatively newly discovered putrescine importer in E. coli (Kurihara et al., 2011). For verification, we tested the role of transporter of PlaP in the presence of exogenous putrescine, spermidine and spermine at the final concentration of $0.1 \mathrm{mM}$, respectively. As expected, exogenous addition of putrescine significantly increased the swimming motility of the complemented strain $\Delta$ speA $\triangle$ potF $\Delta$ plaP $($ plaP $)$, whereas the same treatment had no effect on the motility of triple-deletion mutant $\Delta$ speA $\Delta$ potF $\Delta$ plaP, validating the key role of PlaP in uptake of exogenous putrescine. However, exogenous addition of same concentration of spermidine or spermine had no effect on swimming motility of strain $\Delta$ speA $\Delta$ potF $\Delta$ plaP(plaP $)$ (Supplementary Figure S4). These results are consistent with the data shown in Figure 5, which collectively demonstrate that PotF and PlaP are essential for D. zeae to uptake exogenous putrescine.

\section{Disruption of speA Alters the Intracellular Putrescine Level}

To compare the intracellular concentration of putrescine between wild type $\mathrm{EC} 1$ and mutant $\triangle s p e A$, we derivatized the polyamine molecules in bacterial samples by using benzoyl chloride (Lee et al., 2009; Sethi et al., 2011), and determined the concentration of benzoyled putrescine via high-performance liquid chromatography coupled with mass spectrometry (LCMS). The results showed that the intracellular concentration of putrescine in mutant $\triangle$ speA was about 4.5 -fold lower than that in the wild type EC1 (Figure 6A and Supplementary Figure S5).

To test whether wild type strain could produce and secrete sufficient putrescine signal to induce the swimming motility of the speA mutant $\triangle$ speA, we designed and performed a simple extracellular complementation assay. To a LB plate about $9 \mathrm{~cm}$ in diameter, D. zeae wild type strain EC1 and the mutant $\triangle$ speA were streaked on both ends of the plate and incubated for $12 \mathrm{~h}$ to precondition the medium with extracellular signals. Then a middle section of the plate about $5 \mathrm{~cm}$ in width was removed and replaced with $10 \mathrm{~mL}$ of swimming motility medium. After solidify, mutants $\triangle$ speA and $\Delta$ speA $\triangle$ potF $\triangle$ plaP were streaked respectively on the swimming medium perpendicular to the lines of strain EC1 and mutant $\triangle$ speA on LB medium, and the plates were cultured at $28^{\circ} \mathrm{C}$ for $24 \mathrm{~h}$. As showed in Figure 6B, wild type strain EC1 cells were able to induce the swimming motility of mutant $\Delta$ speA, whereas the mutant $\Delta$ speA cells were unable to induce itself to swim. Importantly, the extracellular signals from wild type strain EC1 could not restore the swimming motility of the mutant $\Delta$ speA $\Delta$ potF $\Delta$ plaP (Figure 6B), in which the pot $F$ and plaP genes encoding putrescine binding proteins of corresponding putrescine transporters were deleted. The above data suggest that deletion of speA abolished the production of signals required for induction of swimming motility in D. zeae, and further validate that the putrescine specific transporters are essential for $D$. zeae to respond to extracellular putrescine signals.

\section{Putrescine Modulates the Transcriptional Expression of Over 200 Genes in D. zeae EC1}

To determine the scope of putrescine signal influence, we examined the global gene expression profiles of wild type strain EC1 and the speA deletion mutant in swimming motility medium with putrescine at the final concentration of $0.1 \mathrm{mM}$ or without using transcriptome analysis. Of the 4,154 genes predicted in EC1 genome (Zhou et al., 2015), expression of 230 genes were significantly (| Fold Change| $\geq 2$-fold, $q \leq 0.005$ ) influenced by putrescine, including 102 genes upregulated and 128 genes downregulated, which were mostly restored by exogenous addition of putrescine (Supplementary Table S3). These putrescine-dependent genes can be grouped into several functional categories, including (i) secretion systems, (ii) transporter systems, (iii) methyl-accepting chemotaxis proteins, (iv) ribosomal proteins, (v) acyltransferase, and (vi) hypothetical proteins (Supplementary Table S3). The reliability of the transcriptome results was verified by semiquantitative RT-PCR analysis of randomly selected genes listed in Supplementary Table S2 and Supplementary Figure S6. Consistent with the findings that putrescine regulated the bacterial motility and biofilm formation, several genes encoding chemotaxis 
A

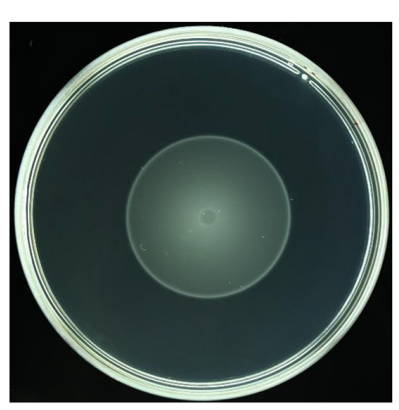

D

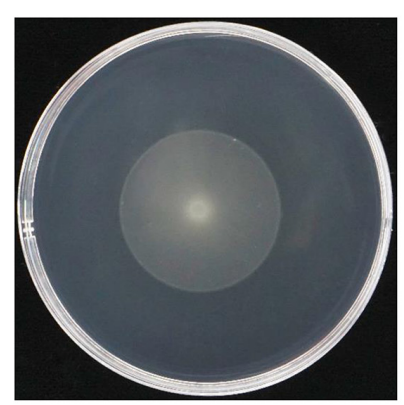

B

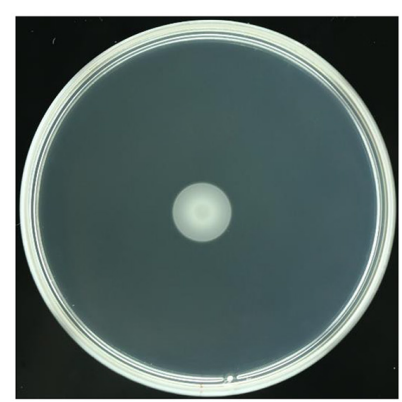

C

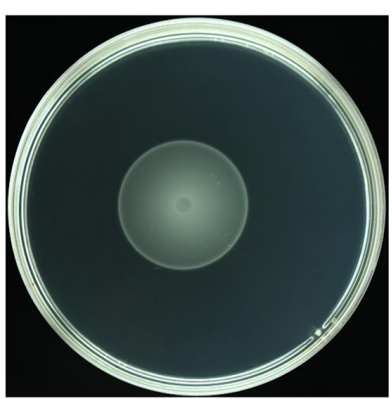

E

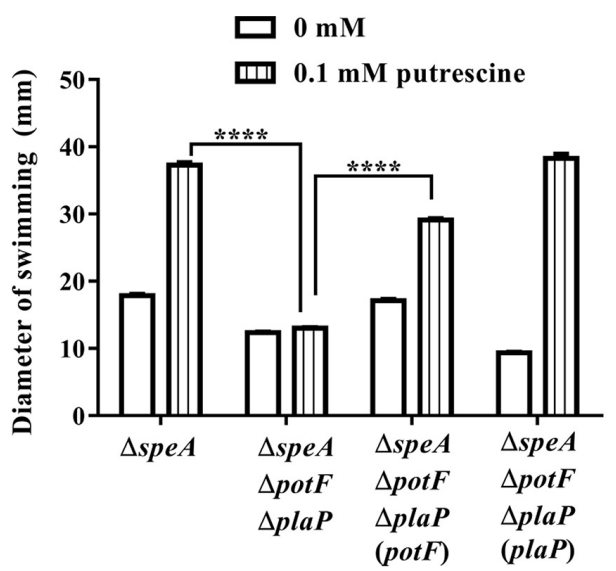

FIGURE 5 | Influence of transporters on putrescine uptaking. The plate bioassay was performed using swimming motility supplemented with 0.1 mM of putrescine for $24 \mathrm{~h}$ at $28^{\circ} \mathrm{C}$. (A) The speA-disrupted mutant; (B) The potF-plaP double disrupted mutant in the speA-disrupted genetic background ( $\Delta$ speA $\Delta$ potF $\Delta$ plaP); (C,D) the complemented strains $\Delta$ speA $\Delta$ potF $\Delta$ plaP(potF) (C) and $\Delta$ speA $\Delta$ potF $\Delta$ plaP(plaP) (D); (E) Quantitative measurement of swimming motility of $\Delta s p e A$ and its derivatives with or without putrescine $(\mathrm{CK})$. The data shown are the means $\pm \mathrm{SE}(n=3) . * * * P<0.0001$.
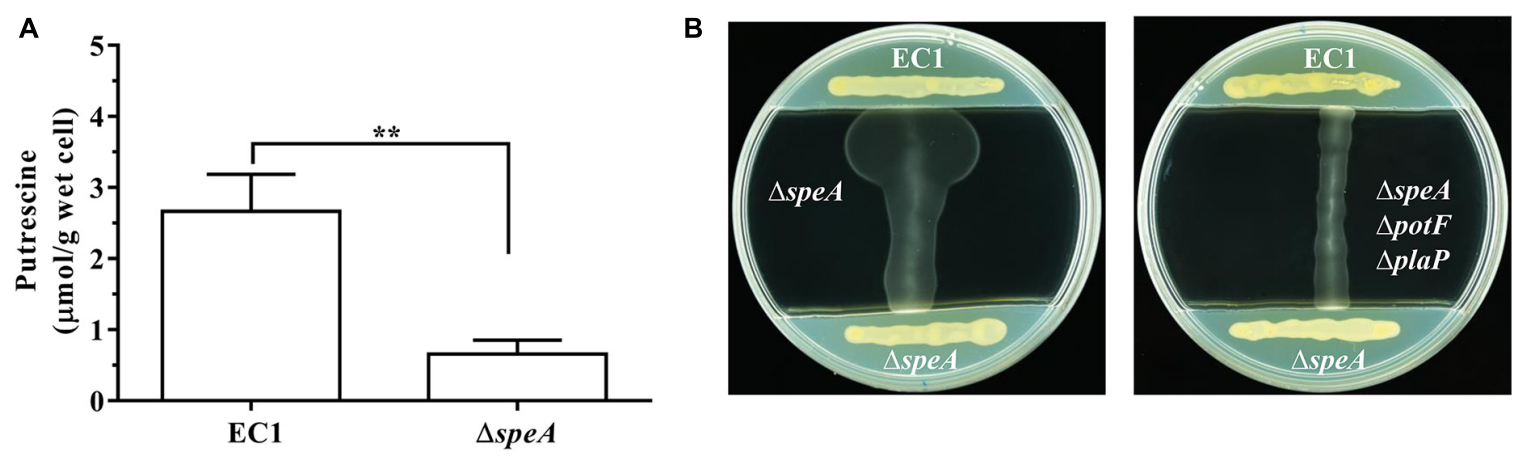

FIGURE 6 | Deletion of speA in D. zeae strain EC1 decreased cellular level of putrescine and abrogated its ability to promote swimming motility. (A) Quantitative measurement of cellular putrescine content in strain EC1 and the speA deletion mutant $\Delta$ speA. The bacterial strains were grown in minimum medium till OD 600 reached about 1.7. Intracellular putrecine molecules were extracted by freezing and thawing with liquid nitrogen, and benzoylated with benzoyl chloride for analysis with LC-MS. The experiment was repeated three times and error bars denote s.e. (B) Putrescine induction of bacterial motility required functional transporters. Strain EC1 and mutant $\triangle$ speA were grown respectively on the opposite ends of LB plate as indicated to precondition the medium. After $12 \mathrm{~h}$, the middle section of the plate was removed and filled with swimming agar medium. Upon solidification, the mutants $\Delta s p e A$ (left panel) and $\Delta$ speA $\Delta$ potF $\Delta$ plaP (right panel) were lined vertically to strain EC1 and the mutant $\Delta$ speA as indicated. The plates were cultured for $24 \mathrm{~h}$ at $28^{\circ} \mathrm{C}$ before photographing. The experiment was repeated three times with similar results. $* * P<0.01$.

and flagellar proteins, which are associated with bacterial motility, were downregulated in the speA deletion mutant but restored by exogenous addition of putrescine (Supplementary
Table S3). In addition, over 10 genes associated with T3SS secretion system were also positively regulated by putrescine (Supplementary Table S3). 


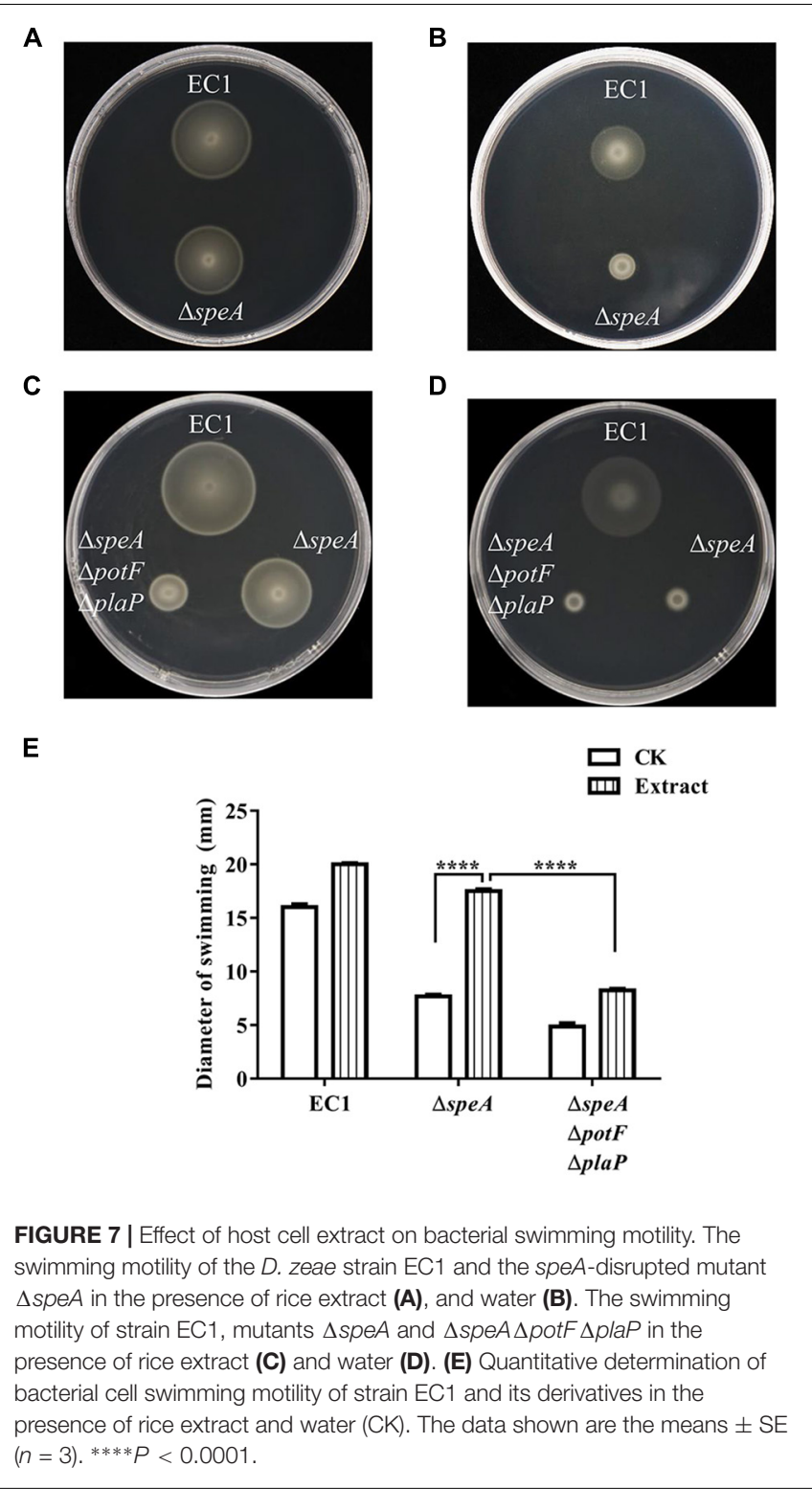

\section{Putrescine Signal Produced by Host Plant Induces the Swimming Motility of D. zeae}

Consistent with the previous report that vigorous growth tissue of plants containing abundant putrescine and other polyamines (Walden et al., 1997), LC-MS analysis indicated that putrescine was present in the rice seedling extract and the total free putrescine concentration was about $27 \mu \mathrm{M}$. We then mixed the extract of rice seedling with semi-preparative medium and measured swimming motility of wild type EC1 and its derivatives. After cultured for $12 \mathrm{~h}$ with rice seedling extract equivalent to $20 \mathrm{mg}$ of seedling tissue per $\mathrm{mL}$ of mixed medium, the swimming motility of the mutant $\Delta s p e A$ was significantly increased by about 2.4-fold in comparison with the blank control in which medium was mixed with water (Figures $7 \mathbf{A}, \mathbf{B}, \mathbf{E}$ ). In contrast, the swimming motility of strain EC1 and mutant $\Delta$ speA $\Delta$ potF $\Delta$ plaP were also increased by host tissue extract but in a much less extent than the speA mutant $\triangle$ speA (Figures $7 \mathrm{C}, \mathbf{D}, \mathbf{E}$ ). The findings indicate that putrescine molecules from host cells could be transported into bacterial cells via putrescine specific transporters and induce the bacterial swimming motility.

\section{Mutation of speA and Transporter Genes in $D$. zeae EC1 Decreases Bacterial Virulence and Invasion Ability on Rice Seeds}

To assess the role of putrescine in modulation of bacterial virulence, rice seeds were incubated with various dilutions of D. zeae strain EC1 and its derivatives, and the rice seed germination rate were measured 5 days after treatment. The results showed that strain EC1 was highly virulent on rice seeds, showing about $88 \%$ inhibition rate when rice seeds were treated with 10 bacterial cells per $\mathrm{mL}$ and total inhibition at 100 bacterial cells per $\mathrm{mL}$ (Figure 8A). Deletion of speA reduced the bacterial virulence by about $35 \%$ at 10 bacterial cells per $\mathrm{mL}$ and about $13 \%$ at 100 bacterial cells (Figure 8A). In contrast, the mutant $\triangle$ speA $\triangle$ potF $\triangle$ plaP, in which both putrescine synthase gene speA and transporter genes pot $F$ and plaP were deleted, was unable to inhibit seed germination at a concentration range from 10 to 1000 bacterial cells per $\mathrm{mL}$, and showed merely about $13 \%$ inhibition rate when the bacterial inoculum was increased to 10,000 cells per $\mathrm{mL}$ (Figure 8A). To test which transporter plays a major role in putrescine-mediated pathogen-host communication, the double deletion mutants $\Delta s p e A \Delta p o t F$ and $\Delta s p e A \Delta p l a P$ were also included in the germination assay. The results clearly indicated that PotF played a dominate role over PlaP in uptaking polyamine signals from host (Figure 8A).

To test how putrescine could influence the bacterial virulence, invasion assay was performed by incubation of rice seeds with the bacterial strains expressing the $g f p$ gene at 100 cells per $\mathrm{mL}$. The inoculated seeds with husk removed were examined by fluorescence microscope, and the bacterial cells retained in rice seeds were isolated and measured at $24 \mathrm{~h}$ after treatment. The results showed that rice seeds treated with strain EC1 displayed higher intensity of fluorescence than those treated with the mutant $\triangle s p e A$, whereas no fluorescence spot was observed in the seeds treated with the triple-deletion mutant $\Delta s p e A \Delta p o t F \Delta$ plaP (Figure 8B). Consistent with the fluorescence analysis, counting bacterial cell numbers invaded into rice seeds showed that on average each seed contained about 1000 strain EC1 cells, less than 500 the mutant $\triangle s p e A$ cells, and none the triple mutant $\Delta$ speA $\Delta$ potF $\Delta$ plaP cells (Figure $\mathbf{8 C}$ ).

\section{DISCUSSION}

Bacterial motility and biofilm are important virulence determinants and survival strategies for microbes to cope with harsh environmental conditions and infecting host organisms. In this study, we showed that the deletion of arginine decarboxylase gene speA in the plant bacterial pathogen $D$. zeae 


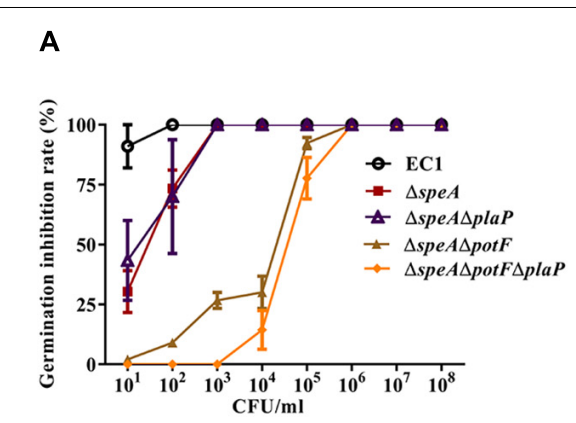

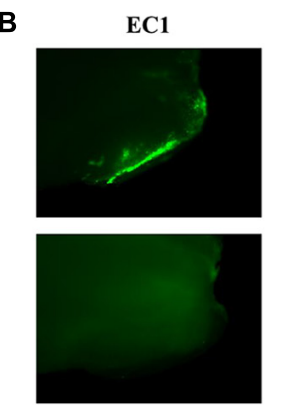

$\triangle$ speA $\triangle$ potF $\triangle$ plaP

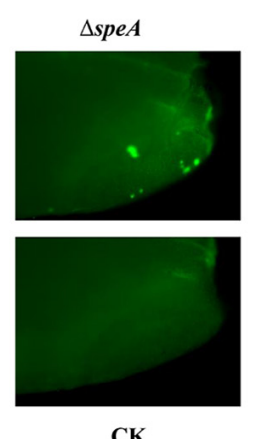

CK

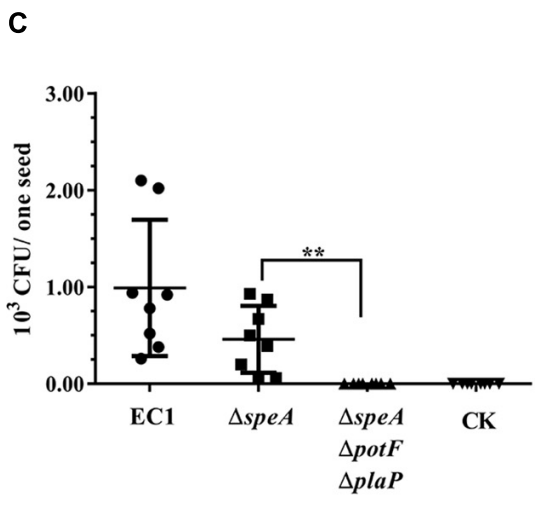

FIGURE 8 | Deletion of speA and putrescine transporters attenuated bacterial invasion and virulence of $D$. zeae EC1 on rice seeds. (A) Rice seed germination assay. Rice seeds were socked in bacterial suspension containing various amounts of bacterial cells as indicated for $5 \mathrm{~h}$ before transferred to plates with moisturized filter papers. The germination rates were counted 5 days later. (B,C) Rice seed invasion assay. Strain EC1 and derivatives were labeled with GFP by in trans expression of the gfp gene. Rice seeds were treated with 100 bacterial cells per $\mathrm{mL}$ for $5 \mathrm{~h}$, and then incubated for $24 \mathrm{~h}$ at $28^{\circ} \mathrm{C}$. The husks of rice seeds were removed for examination under a fluorescence microscope (B) and for bacterial CFU counting (C). The representative photographs were presented and the data shown are the means $\pm \mathrm{SE}(n=3)$. ${ }^{* *} P<0.01$.

significantly handicapped its swimming and swarming motility, and drastically attenuated the bacterial biofilm formation ability (Figure 1). The defective phenotypes of the speA mutant could be rescued via in trans expression of speA in mutant or exogenous addition of putresciene or by wild type D. zeae EC1 grown at vicinity (Figures 1-4, 6B). This is agreeable with the finding that disruption of speA resulted in a drastic reduction of intracellular putrescine level (Figure 6A). Furthermore, we presented evidence that there was no correlation between the defective phenotypes and growth rate, as the growth rate of speA mutant was similar to wild type in the defined minimal medium with no polyamines (Supplementary Figure S2). These results demonstrated that SpeA is essential for putrescine biosynthesis and putrescine is required for bacterial cell motility and biofilm formation in D. zeae. A similar role of putrescine in regulation of bacterial motility or biofilm formation has also been reported in human bacterial pathogens P. mirabilis (Sturgill and Rather, 2004), Y. pesti (Patel et al., 2006; Wortham et al., 2010), and environmental isolate S. oneidensis (Ding et al., 2014). The identified roles of putrescine in the plant pathogen $D$. zeae thus further validate its conserved role in modulation of bacterial motility and biofilm formation in diverse microorganisms. The data of transcriptome analysis suggest that putrescine might modulate bacterial motility through regulation of bacterial chemotaxis and flagellar biogenesis (Supplementary Table S3), as bacterial movement is guided and driven by chemotaxis and flagellar, respectively (Guttenplan and Kearns, 2013; Jones and Armitage, 2015).

Bacteria require either a polyamine influx transporter or a sensor when extracellular polyamines are used as growth factors or signaling molecules (Furuchi et al., 1991; Karatan et al., 2005; Zhou et al., 2007). This is because polyamines are hydrophilic molecules that cannot passively diffuse across that bacterial plasma membrane. In this work, we found two putrescine specific transporters in $D$. zeae strain EC1, represented by their cognate putrescine binding proteins $\mathrm{PlaP}$ and PotF, respectively, which collectively play key roles for influx transportation of exogenous putrescine into bacterial cells (Figures 5, 8 and Supplementary Figure S3). Significantly, our results showed unequivocally that two transporters, in particular the one represented by PotF, are essential for influxing extracellular putrescine signals either from D. zeae itself or from host plant (Figures 6-8). Identification of two putrescine influx transporters further validates the important role of putrescine as a signal for bacterial cell-cell communication and for pathogen-host interactions.

Interestingly, two transporters appeared to act differently in modulation of bacterial motility when assayed in the presence or absence of exogenous putrescine. In the absence of exogenous putrescine, deletion of potF in the genetic background of speA mutant resulted in reduced bacterial swimming motility to a level similar to the speA/potF/plaP triple-deletion mutant $\triangle$ speA $\triangle$ potF $\triangle$ plaP, whereas further deletion of plaP in the mutant $\triangle s p e A$ caused only a minor reduction in motility compared to its parental strain $\triangle s p e A$ (Supplementary Figure S3). This suggests that PotF transporter plays a dominant role in regulation of bacterial biological motility. In contrast, however, in the presence of exogenous putrescine, complementation of the speA/potF/plaP tripledeletion mutant $\triangle$ speA $\triangle$ potF $\triangle$ plaP with plaP could fully restore the bacterial motility in a capacity even superior than potF (Figure 5). Similarly, motility assay of doubledeletion mutants $\triangle s p e A \Delta p o t F$ and $\triangle$ speA $\Delta p l a P$ further confirmed the superior performance of PlaP transporter over PotF with exogenous addition of putrescine (Supplementary Figure S3E). The contrasting performance of PotF and PlaP transporters with or without exogenous putrescine may suggest different sensitivities of two transporters to putrescine, either at transcriptional or translational level or both, which awaits further investigations.

In addition to its role as a bacterial intercellular signal, the results from this study also indicate that putrescine plays a key role in pathogen-host interactions. Putrescine is present 
in both bacterial and plant cells (Igarashi and Kashiwagi, 2000), but it is not clear whether its level in plant cells is sufficiently high enough to influence the physiological processes of bacterial cells. By using a combination of putrescine derivatization and HPLC-MS analysis, we showed that rice seeds in germination contained about $27 \mu \mathrm{M}$ putrescine. Given that exogenous addition of $10 \mu \mathrm{M}$ putrescine could substantially induce bacterial swimming motility (Figure 3A), we argued that $D$. zeae could exploit the putrescine molecules produced by rice host as a cross-kingdom communication signal to promote bacterial motility and systemic infection. The notion is greatly strengthened by the observation that deletion of the putrescine binding proteins of cognate transporters significantly reduced the host cell extract-induced swimming motility (Figure 7C). The role of putrescine in host-pathogen interaction is reminiscent to the findings of spermidine in the human bacterial pathogen $P$. aeruginosa. It was shown previously that the spermidine produced by mammalian host could be exploited by $P$. aeruginosa to trigger the transcriptional expression of type III secretion system (T3SS) genes via a polyamine transporter (Zhou et al., 2007), although it remains to be determined whether $P$. aeruginosa could also produce sufficient amount of spermidine molecules and utilize these molecules as an intercellular signal to influence T3SS expression. Similarly, transcriptome analysis indicated that putrescine might also play a role in regulation of T3SS expression in D. zeae EC1 (Supplementary Table S3), which awaits further investigations. The findings from this study add one more polyamine molecule as an effective and potent cross-kingdom communication signal in modulation of bacterial physiological activity. It remains to be investigated whether host could induce putrescine biosynthesis in $D$. zeae or the bacterial infection might trigger host plant to produce higher level of putrescine than the untreated rice seeds. A recent study showed that putrescine was enriched 76 -fold to $37 \mu \mathrm{M}$ in $R$. solanacearuminfected sap of tomato xylem vessels, and treatment of plants with $500 \mu \mathrm{M}$ putrescine before inoculation accelerated wilt symptom development and bacterial growth and spread (Lowe-Power et al., 2018). Consistent with the latter option, the same study also showed that $R$. solanacearum infection induced expression of several plant putrescine biosynthesis genes (Lowe-Power et al., 2018).

Cell motility is closely associated with bacterial pathogenesis and virulence, which has been documented in Ralstonia solanacearum (Tans-Kersten et al., 2001), and Proteus mirabilis (Allison et al., 1994; Kurihara et al., 2013). Given this important function, it seems highly logic and rational that $D$. zeae has involved mechanisms allowing utilization of putrescine signals from bacterium itself and host plant in modulation of virulence. A clear cumulative effect of putrescine biosynthesis and uptaking on bacterial motility and virulence was shown when speA single deletion mutant and speA/potF douple mutant and speA/potF/plaP triple mutant were compared (Supplementary Figure S3E and Figure 8A). Interestingly, deletion of speA and its transporter genes potF and plaP did not affect biosynthesis of zeamines (data not shown), which are known phytotoxins and inhibitors on rice seed germination (Zhou et al., 2011; Cheng et al., 2013). To explore the mechanism that links motility and virulence, we used D. zeae EC1 and its derivatives expressing $g f p$ to infect rice seeds, and the results showed that the pathogen failed to penetrate and invade rice seeds when putrescine production and transportation were blocked (Figures $\mathbf{8 B}, \mathbf{C}$ ). This suggests that for optimum toxic effect, phytotoxin zeamines should be produced inside rice seeds. Intriguingly, D. zeae produces only a low level of zeamines about $7 \mu \mathrm{M}$ per $\mathrm{mL}$ in minimum medium (Liao et al., 2014), but total inhibition of rice seed germination requires a high concentration of purified zeamines ( $>480 \mu \mathrm{M}$ ) (Zhou et al., 2011). This is apparently inconsistent with the finding that inoculation with only a few bacterial cells (about 10 cells per $\mathrm{mL}$ ) could abrogate rice seed germination (Figure 8A; Hussain et al., 2008). The results from this study explain this intriguing puzzle and provide useful clues and new targets for designing and developing new approaches to prevent and control bacterial infections.

\section{DATA AVAILABILITY}

This manuscript contains previously unpublished data. The raw data of transcriptome analyses were available under SRA accession number PRJNA516290.

\section{AUTHOR CONTRIBUTIONS}

$\mathrm{L}-\mathrm{HZ}$ and $\mathrm{ZS}$ conceived and designed the experiments. ZS, QW, YL, and ZL performed the experiments. LX, JZ, and ZC analyzed the data. ZS and L-HZ wrote the manuscript. All authors read and approved the final manuscript.

\section{FUNDING}

This work was financially supported by the grants from the National Basic Research Program of China (973 Program, Grant No. 2015CB150600), the National Natural Science Foundation of China (Grant No. 31330002), Key Projects of Guangzhou Science and Technology Plan (Grant No. 201804020066), and Guangdong Technological Innovation Strategy of Special Funds (Grant No. 2018B020205003).

\section{ACKNOWLEDGMENTS}

We gratefully acknowledge the Novogene (Beijing, China) for their help in transcriptome analyses.

\section{SUPPLEMENTARY MATERIAL}

The Supplementary Material for this article can be found online at: https://www.frontiersin.org/articles/10.3389/fmicb.2019. 01950/full\#supplementary-material 


\section{REFERENCES}

Allison, C., Emödy, L., Coleman, N., and Hughes, C. (1994). The role of swarm cell differentiation and multicellular migration in the uropathogenicity of Proteus mirabilis. J. Infect. Dis. 169, 1155-1158. doi: 10.1093/infdis/169.5.1155

Barras, F., Van Gijsegem, F., and Chatterjee, A. K. (1994). Extracellular enzymes and pathogenesis of soft-rot Erwinia. Annu. Rev. Phytopathol. 32, 201-234. doi: 10.1146/annurev.py.32.090194.001221

Boyle, S. M., Markham, G. D., Hafner, E. W., Wright, J. M., Tabor, H., and Tabor, C. W. (1984). Expression of the cloned genes encoding the putrescine biosynthetic enzymes and methionine adenosyltransferase of Escherichia coli (SpeA, SpeB, SpeC, and MetK). Gene 30, 129-136. doi: 10.1016/0378-1119(84) 90113-6

Cheng, Y. Y., Liu, X. L., An, S. W., Chang, C. Q., Zou, Y. Q., Huang, L. H., et al. (2013). A nonribosomal peptide synthase containing a stand-alone condensation domain is essential for phytotoxin zeamine biosynthesis. Mol. Plant Microbe. Interact. 26, 1294-1301. doi: 10.1094/MPMI-04-13-0098-R

Collmer, A., and Bauer, D. W. (1994). Erwinia chrysanthemi and Pseudomonas syringae: plant pathogens trafficking in extracellular virulence proteins. Curr. Top. Microbiol. 192, 43-78. doi: 10.1007/978-3-642-78624-2_3

Ding, Y. Z., Peng, N., Du, Y. H., Ji, L. H., and Cao, B. (2014). Disruption of putrescine biosynthesis in Shewanella oneidensis enhances biofilm cohesiveness and performance in Cr(VI) immobilization. Appl. Environ. Microbiol. 80, 14981506. doi: 10.1128/AEM.03461-13

Furuchi, T., Kashiwagi, K., Kobayashi, H., and Igarashi, K. (1991). Characteristics of the gene for a spermidine and putrescine transport system that maps at 15 min on the Escherichia coli chromosome. J. Biol. Chem. 266, 20928-20933.

Goto, M. (1979). Bacterial foot rot of rice caused by a strain of Erwinia chrysanthemi. Phytopathology 69, 213-216.

Guttenplan, S. B., and Kearns, D. B. (2013). Regulation of flagellar motility during biofilm formation. FEMS Microbiol. Rev. 37, 849-871. doi: 10.1111/1574-6976. 12018

Hussain, M. B. B. M., Zhang, H. B., Xu, J. L., Liu, Q., Jiang, Z., and Zhang, L. H. (2008). The acyl-homoserine lactone-type quorum-sensing system modulates cell motility and virulence of Erwinia chrysanthemi pv. zeae. J. Bacteriol. 190, 1045-1053. doi: 10.1128/JB.01472-07

Igarashi, K., and Kashiwagi, K. (2000). Polyamines: mysterious modulators of cellular functions. Biochem. Biophys. Res. Commun. 271, 559-564. doi: 10.1006/ bbrc. 2000.2601

Jones, C. W., and Armitage, J. P. (2015). Positioning of bacterial chemoreceptors. Trends Microbiol. 23, 247-256. doi: 10.1016/j.tim.2015. 03.004

Karatan, E., Duncan, T. R., and Watnick, P. I. (2005). NspS, a predicted polyamine sensor, mediates activation of Vibrio cholerae biofilm formation by norspermidine. J. Bacteriol. 187, 7434-7443. doi: 10.1128/JB.187.21.7434-7443. 2005

Kashiwagi, K., and Igarashi, K. (1988). Adjustment of polyamine contents in Escherichia coli. J. Bacteriol. 170, 3131-3135. doi: 10.1128/jb.170.7.3131-3135. 1988

Kashiwagi, K., Miyanoto, S., Suzuki, F., Kobayashi, H., and Igarashi, K. (1992). Excretion of putrescine by the putrescine-ornithine antiporter encoded by the potE gene of Escherichia coli. Proc. Natl. Acad. Sci. U.S.A. 89, 4529-4533. doi: 10.1073/pnas.89.10.4529

Kurihara, S., Oda, S., Tsuboi, Y., Kim, H. G., Oshida, M., Kumagai, H., et al. (2008). $\gamma$-Glutamylputrescine synthetase in the putrescine utilization pathway of Escherichia coli K-12. J. Biol. Chem. 283, 19981-19990. doi: 10.1074/jbc. M800133200

Kurihara, S., Sakai, Y., Suzuki, H., Muth, A., Phanstiel, O. IV, and Rather, P. N. (2013). Putrescine importer PlaP contributes to swarming motility and urothelial cell invasion in Proteus mirabilis. J. Biol. Chem. 288, 15668-15676. doi: 10.1074/jbc.M113.454090

Kurihara, S., Suzuki, H., Oshida, M., and Benno, Y. (2011). A novel putrescine importer required for type 1 pili-driven surface motility induced by extracellular putrescine in Escherichia coli K-12. J. Biol. Chem. 286, 10185-10192. doi: 10. 1074/jbc.M110.176032

Kurihara, S., Tsuboi, Y., Oda, S., Kim, H. G., Kumagai, H., and Suzuki, H. (2009). The putrescine importer PuuP of Escherichia coli K-12. J. Bacteriol. 191, 2776-2782. doi: 10.1128/JB.01314-08
Lee, J., Sperandio, V., Frantz, D. E., Longgood, J., Camilli, A., Phillips, M. A., et al. (2009). An alternative polyamine biosynthetic pathway is widespread in bacteria and essential for biofilm formation in Vibrio cholerae. J. Biol. Chem. 284, 9899-9907. doi: 10.1074/jbc.M900110200

Liao, L. S., Cheng, Y. Y., Liu, S. Y., Zhou, J. N., An, S. W., Lv, M. F., et al. (2014). Production of novel antibiotics zeamines through optimizing Dickeya zeae fermentation conditions. PLoS One 9:e116047. doi: 10.1371/journal.pone. 0116047

Lowe-Power, T. M., Hendrich, C. G., Roepenack-Lahaye, E. V., Li, B., Wu, D. S., Mitra, R., et al. (2018). Metabolomics of tomato xylem sap during bacterial wilt reveals Ralstonia solanacearum produces abundant putrescine, a metabolite that accelerates wilt disease. Environ. Microbiol. 20, 1330-1349. doi: 10.1111/14622920.14020

Nassar, A., Bertheau, Y., Dervin, C., Narcy, J. P., and Lemattre, M. (1994). Ribotyping of Erwinia chrysanthemi strains in relation to their pathogenic and geographic distribution. Appl. Environ. Microbiol. 60, 3781-3789. doi: 10.1016/ 0027-5107(94)90118-X

Patel, C. N., Wortham, B. W., Lines, J. L., Fetherston, J. D., Perry, R. D., and Oliveira, M. A. (2006). Polyamines are essential for the formation of plague biofilm. J. Bacteriol. 188, 2355-2363. doi: 10.1128/jb.188.7.2355-2363.2006

Pistocchi, R., Kashiwagi, K., Miyamoto, S., Nukui, E., Sadakata, Y., Kobayashi, H., et al. (1993). Characteristics of the operon for a putrescine transport system that maps at 19 minutes on the Escherichia coli chromosome. J. Biol. Chem. 268, $146-152$.

Sethi, R., Chava, S. R., Bashir, S., and Castro, M. E. (2011). An improved high performance liquid chromatographic method for identification and quantization of polyaminesas benzoylated derivatives. Am. J. Analyt. Chem. 2, 456-469. doi: 10.4236/ajac.2011.24055

Sinha, S. K., and Prasad, M. (1977). Bacterial stalk rot of maize, its symptoms and host-range. Zentralbl Bakteriol Parasitenkd Infektkrankh Hyg. 132, 81-88. doi: 10.1016/s0044-4057(77)80037-3

Sturgill, G., and Rather, P. N. (2004). Evidence that putrescine acts as an extracellular signal required for swarming in Proteus mirabilis. Mol. Microbiol. 51, 437-446. doi: 10.1046/j.1365-2958.2003.03835.x

Tabor, C. W., and Tabor, H. (1987). The speE speD operon of Escherichia coli. Formation and processing of a proenzyme form of s-adenosyltransferase decarboxylase. J. Biol. Chem. 262, 16037-16040. doi: 10.1109/9. 661596

Tabor, C. W., Tabor, H., and Xie, Q. W. (1986). Spermidine synthase of Escherichia coli localization of the speE gene. Proc. Natl. Acad. Sci. U.S.A. 83, 6040-6044. doi: $10.1073 /$ pnas.83.16.6040

Tans-Kersten, J., Huang, H., and Allen, C. (2001). Ralstonia solanacearum needs motility for invasive virulence on tomato. J. Bacteriol. 183, 3597-3605. doi: 10.1128/JB.183.12.3597-3605.2001

Vassylyev, D. G., Tomitori, H., Kashiwagi, K., Morikawa, K., and Igarashi, K. (1998). Crystal structure and mutational analysis of the Escherichia coli putrescine receptor. Structural basis for substrate specificity. J. Biol. Chem. 273, 17604-17609. doi: 10.1074/jbc.273.28.1760

Wagner, G. P., Kin, K., and Lynch, V. J. (2012). Measurement of mRNA abundance using RNA-seq data: RPKM measure is inconsistent among samples. Theory Biosci. 131, 281-285. doi: 10.1007/s12064-012-0162-3

Walden, R., Cordeiro, A., and Tiburcio, A. F. (1997). Polyamines: small molecules triggering pathways in plant growth and development. Plant Physiol. 113, 1009-1013. doi: 10.1104/pp.113.4.1009

Wang, L., Feng, Z., Wang, X., Wang, X., and Zhang, X. (2010). DEGseq: an $\mathrm{R}$ package for identifying differentially expressed genes from RNA-seq data. Bioinformatics 26, 136-138. doi: 10.1093/bioinformatics/btp612

Wortham, B. M., Oliveira, M. A., Fetherston, J. D., and Perry, R. D. (2010). Polyamines are required for the expression of key Hms proteins important for Yersinia pestis biofilm formation. Environ. Microbiol. 12, 2034-2047. doi: 10.1111/j.1462-2920.2010.02219.x

Wu, D. H., Lim, S. C., Dong, Y. H., Wu, J. E., Tao, F., Zhou, L., et al. (2012). Structural basis of substrate binding specificity revealed by the crystal structures of polyamine receptors SpuD and SpuE from Pseudomonas aeruginosa. J. Mol. Biol. 416, 697-712. doi: 10.1016/j.jmb.2012. 01.010

Wu, J., Zhang, H. B., Xu, J. L., Cox, R. J., Simpson, T. J., and Zhang, L. H. (2010). 13C Labeling reveals multiple amination reactions in the biosynthesis of a novel 
polyketide polyamine antibitic zeamine from Dickeya zeae. Chem. Commun. 46, 333-335. doi: 10.1039/B916307G

Zhang, J. X., Lin, B. R., Shen, H. F., and Pu, X. M. (2013). Genome sequence of the banana pathogen Dickeya zeae strain MS1, which causes bacterial soft rot. Genome Announc. 1, e317-13. doi: 10.1128/genomeA. 00317-13

Zhou, J. N., Cheng, Y. Y., Lv, M. F., Liao, L. S., Chen, Y. F., Gu, Y. F., et al. (2015). The complete genome sequence of Dickeya zeae EC1 reveals substantial divergence from other Dickeya strains and species. BMC Genomics 16:571. doi: 10.1186/s12864-015-1545-x

Zhou, J. N., Zhang, H. B., Lv, M. F., Chen, Y. F., Liao, L. S., Cheng, Y. Y., et al. (2016). SlyA regulates phytotoxin production and virulence in Dickeya zeae EC1. Mol. Plant Pathol. 17, 1398-1408. doi: 10.1111/mpp. 12376

Zhou, J. N., Zhang, H. B., Wu, J., Liu, Q., Xi, P., Lee, J., et al. (2011). A novel multidomian polyketide synthase is essential for zeamine production and the virulence of Dickeya zeae. Mol.
Plant Microbe. Interact. 24, 1156-1164. doi: 10.1094/MPMI-04-110087

Zhou, L., Wang, J., and Zhang, L. H. (2007). Modulation of bacterial type III secretion system by a spermidine transporter dependent signaling pathway. PLoS One 2:e1291. doi: 10.1371/journal.pone.0001291

Conflict of Interest Statement: The authors declare that the research was conducted in the absence of any commercial or financial relationships that could be construed as a potential conflict of interest.

Copyright (๑) 2019 Shi, Wang, Li, Liang, Xu, Zhou, Cui and Zhang. This is an open-access article distributed under the terms of the Creative Commons Attribution License (CC BY). The use, distribution or reproduction in other forums is permitted, provided the original author(s) and the copyright owner(s) are credited and that the original publication in this journal is cited, in accordance with accepted academic practice. No use, distribution or reproduction is permitted which does not comply with these terms. 